\title{
Infective endocarditis in a 52-year-old male patient with vegetations of all four heart valves and extremely high cardiac operative risk
}

\author{
Paweł Maeser, Robert Morawiec, Jarosław Drożdż \\ $2^{\text {nd }}$ Department of Cardiology, Chair of Cardiology, Cardiac Surgery and Vascular Diseases, Medical University of Lodz, Łódź, Poland
}

\author{
Correspondence to: \\ Robert Morawiec, MD \\ $2^{\text {nd }}$ Department of Cardiology, \\ Chair of Cardiology, Cardiac \\ Surgery and Vascular Diseases, \\ Medical University of Lodz, \\ Central Clinical Hospital, \\ Pomorska 251, 90-213 Łódź, \\ Poland, \\ phone: + 48422014308 , \\ e-mail: \\ robert.morawiec@umed.lodz.pl \\ Copyright by the Author(s), 2022 \\ DOI: 10.33963/KP.a2021.0191 \\ Received: \\ November 20, 2021 \\ Accepted: \\ December 28, 2021 \\ Early publication date: \\ December 29, 2021
}

A 52-year-old male presenting with weakness, weight loss, and low-grade fever, which had been worsening over the past 6 months, was re-admitted to the district hospital after 6 inconclusive hospitalizations in at least 6 different hospitals and emergency departments.

Transthoracic echocardiography (TTE) showed massive vegetations of the aortic, mitral, and pulmonary valves. Physical examination and medical history revealed highly advanced dental caries as the only significant risk factor of infective endocarditis (IE) in this case. Positive blood culture with Staphylococcus hominis and Streptococcus gallolyticus was obtained. During hospitalization, the patient got retinal artery occlusion caused by embolic material separated from vegetations. After transferring the patient to our department, laboratory findings revealed moderate anemia, exacerbation of chronic kidney disease, and significantly increased cardiac injury markers. On TTE, the vegetations (Figure $1 \mathrm{~A}-\mathrm{C}$ ) were accompanied by severe mitral and tricuspid regurgitation, moderate aortic and pulmonary regurgitation, enlargement of heart cavities, and a high probability of aortic, pulmonary, and mitral cusps perforation (Figure 1D). Despite the standard treatment including acute heart failure management and targeted antibiotic therapy, due to gradual worsening of the patient's clinical condition, the Heart Team decided against transesophageal echocardiography and disqualified the patient from surgery due to an extremely high cardiac operative risk (EuroSCORE II >40\%). The patient died on the $4^{\text {th }}$ day of hospitalization.

Infective endocarditis is a rare condition with only $1.7-7.9$ cases per 100000 and occurs most commonly on a single valve [1]. Our case, with the abnormalities of 4 native valves and the initial signs dating back 6 months prior to hospitalization, is exceptionally unique.

Heterogeneous clinical presentation creates significant diagnostic challenges in patients with infective endocarditis. Fever $>38^{\circ} \mathrm{C}$ as an isolated symptom occurs in $83.4 \%$ of cases, whereas other symptoms, including new cardiac murmurs, fatigue, or weight loss are not IE-specific, are often correlated to the localization of IE, and occur in less than $40 \%$ of patients [1]. Risk factors associated with IE, like prosthetic valves, drugs injection, intracardiac devices, and others, can help to identify a group of patients with a higher probability of $\mathrm{IE}$, which on average has a mortality rate of $20 \%-25 \%$ [2].

Although valvular surgery of patients with IE is associated with lower 1-year mortality [3], sometimes, due to many clinical risk co-factors, the surgical risk is too high. Mitral valve surgery and multiple valve surgery significantly increase mortality [4]. EuroSCORE II helps to predict surgical risk in cardiac surgery. In the presented case the calculated risk of $40 \%$ was the result of active endocarditis, renal impairment, the urgency of the procedure, and the extent of the intervention. Such a high score is rarely seen and indicates that the operation is unreasonable.

One of the most crucial steps in managing IE is a quick implementation of antibiotics and surgical treatment, as both of these interventions have a reduced effectiveness with time. The severe medical condition of our patient was the outcome of a late diagnosis and delayed treatment. Close medical supervision 

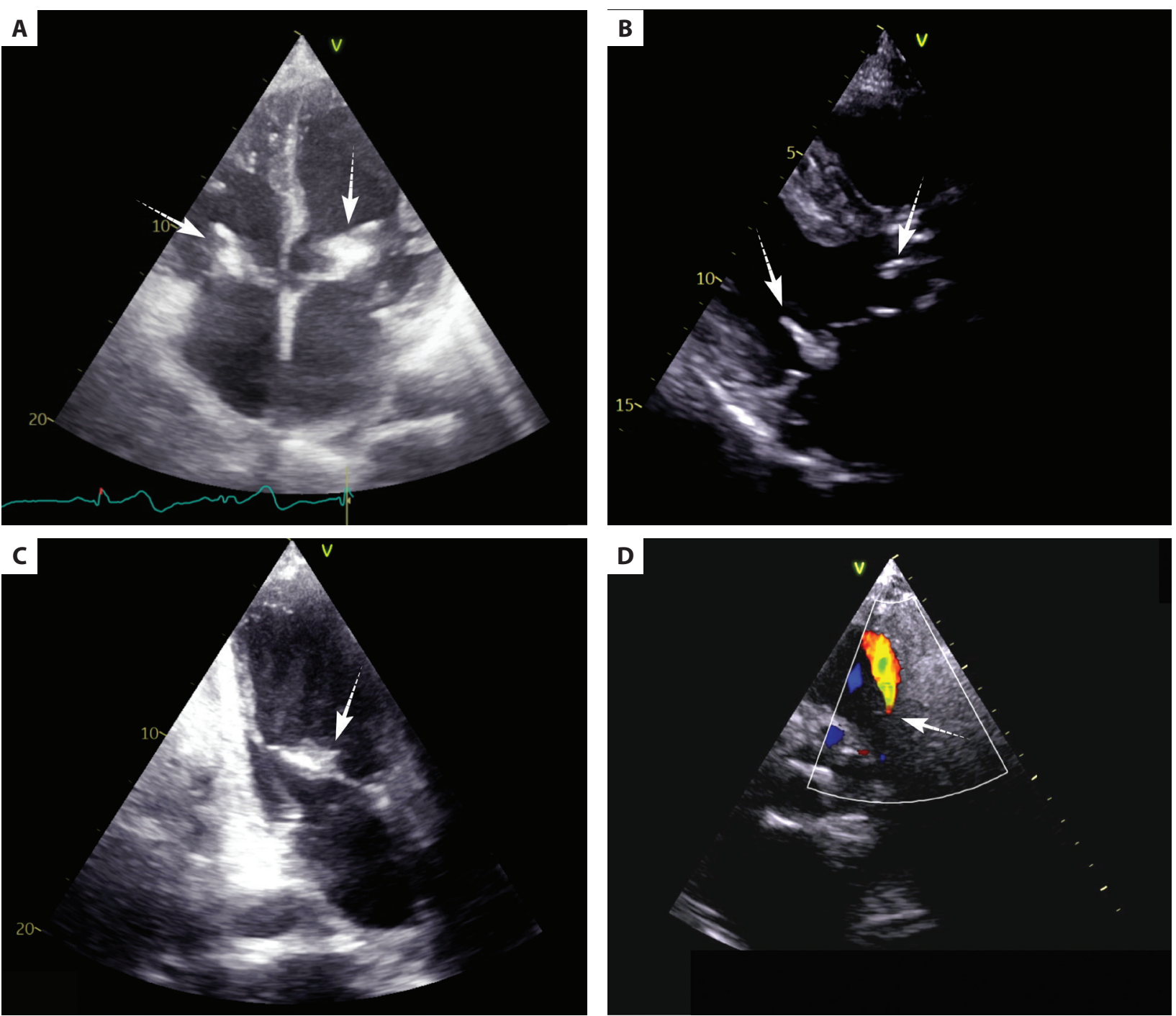

Figure 1. A. Transthoracic echocardiography, apical 4-chamber view. Vegetations of mitral and tricuspid valves have been marked by the arrows. B. Transthoracic echocardiography, parasternal long-axis view. Massive vegetations of mitral and aortic valves have been marked by the arrows. C. Transthoracic echocardiography, apical 2-chamber view. Massive vegetation of the mitral valve has been marked by the arrow. D. Transthoracic echocardiography, parasternal short-axis view with color Doppler. Cusp perforation of the pulmonary valve has been marked by the arrow

can help prevent the development of IE as massive as in the discussed case. The role of the multidisciplinary Heart Team is to make a quick and rational decision, based on the risk assessment, factors not included in common scales (such as EuroSCORE II), and the general condition of the patient.

\section{Article information}

Conflict of interest: None declared.

Open access: This article is available in open access under Creative Common Attribution-Non-Commercial-No Derivatives 4.0 International (CC BY-NC-ND 4.0) license, allowing to download articles and share them with others as long as they credit the authors and the publisher, but without permission to change them in any way or use them commercially. For commercial use, please contact the journal office at kardiologiapolska@ptkardio.pl.

\section{REFERENCES}

1. Muñoz P, Kestler M, De Alarcon A, et al. Spanish Collaboration on Endocarditis-Grupo de Apoyo al Manejo de la Endocarditis Infecciosa en España (GAMES). Current epidemiology and outcome of infective endocarditis: a multicenter, prospective, cohort study. Medicine (Baltimore). 2015; 94(43): e1816, doi: 10.1097/MD.0000000000001816, indexed in Pubmed: 26512582.

2. Cuervo G, Escrihuela-Vidal F, Gudiol C, et al. Current challenges in the management of infective endocarditis. Front Med (Lausanne). 2021; 8: 641243, doi: 10.3389/fmed.2021.641243, indexed in Pubmed: 33693021.

3. Kiefer T, Park L, Tribouilloy $C$, et al. Association between valvular surgery and mortality among patients with infective endocarditis complicated by heart failure. JAMA. 2011; 306(20): 2239-2247, doi: 10.1001/jama.2011.1701, indexed in Pubmed: 22110106.

4. Østergaard L, Smerup MH, Iversen $\mathrm{K}$, et al. Differences in mortality in patients undergoing surgery for infective endocarditis according to age and valvular surgery. BMC Infect Dis. 2020; 20(1): 705, doi: 10.1186/s12879020-05422-8, indexed in Pubmed: 32977755. 